\title{
EL DESARROLLO DE DATOS DE CALIDAD Y EL CAMBIO TÉRMICO OBSERVADO EN ESPAÑA
}

\author{
Javier Sigro, Manola Brunet y Enric Aguilar \\ Centro en Cambio Climático (C3) de la Universitat Rovira i Virgili
}

\section{RESUMEN}

El objetivo de este trabajo es ofrecer una metodología para el desarrollo de bases de datos de largo recorrido de temperatura diaria ajustada, reflexionando sobre la dificultad en recabar datos climáticos históricos e información sobre ellos, las causas más habituales de sesgo en las series de temperatura diaria españolas, las metodologías empleadas con éxito en la detección de errores y rupturas de homogeneidad de las series y las herramientas desarrolladas por los autores para su minimización. A partir de los datos ajustados se construyen series regionales de temperatura y se detallan las significativas tendencias al calentamiento detectadas en la temperatura media, máxima y mínima diaria española, intensificadas en las últimas décadas.

\begin{abstract}
The goal of this paper is to offer a methodology for the development of long-term daily adjusted temperature datasets. We discuss about the problems related with the compilation of historical climate data, the most common biases impacting the series of daily temperatures in Spain and the successful methods applied to the detection of inhomogeneities and the tools developed for the minimization of their effect over the time series. After this, we construct regional series from the adjusted data and study and detail the significant trends found in mean, maximum and minimum daily temperature series for the last decades in Spain.
\end{abstract}

\section{Introducción}

Los registros climáticos instrumentales históricos de alta calidad constituyen una pieza clave para la realización de estudios robustos, consistentes y fiables que permitan la mejor detección, comprensión, predicción y respuesta a los impactos asociados a la variabilidad y cambio climático. La obtención de bases de datos climáticos de alta calidad y alta resolución temporal y espacial es esencial para los estudios de detección y atribución del 
cambio climático, la calibración de datos de satélite o la generación de productos en malla y de re-análisis, además de permitir el desarrollo de las respuestas más apropiadas en las estrategias de adaptación y mitigación.

Los registros instrumentales se remontan en muchas regiones del planeta hasta el siglo XIX, llegando en unas pocas ocasiones hasta el siglo XVII. Estos registros climáticos históricos se han obtenido en múltiples emplazamientos y con diferentes escalas temporales, desde diarias y subdiarias a datos mensuales.

La necesidad de un mejor conocimiento de la variabilidad climática y el cambio a largo plazo del clima, sus factores de forzamiento y los impactos sobre el medio natural y humano, obliga a los investigadores del clima a generar bases de datos de largo recorrido y alta calidad. Sin embargo, uno de los principales obstáculos se halla en la todavía menguada disponibilidad y accesibilidad a datos de calidad, especialmente para escalas menores que la global. En muchos casos la información esta dispersa y fragmentada, no existen registros centralizados de ella y se encuentra en soportes no directamente utilizables para la investigación. No se trata tanto de una carencia de datos como de fondos, tanto a escala local o nacional como a escala regional o internacional, que permitan disponer de los recursos humanos y técnicos necesario para recuperar los datos observacionales del pasado y transferirlos a formatos digitales fácilmente utilizables por las herramientas de análisis computacional actual.

Incluso con la dotación económica suficiente para emprender esta tarea, diversos son los pasos que deben considerarse a la hora de diseñar el desarrollo de una base de datos históricos o de largo recorrido.

En primer lugar, son necesarias misiones de rescate de datos destinadas a la localización, recuperación, preservación y transferencia al formado digital de los datos climáticos antiguos, según recomendaciones de la Organización Meteorológica Mundial (OMM) en recientes guías publicadas (Brunet et al., 2008; Tan et al., 2004).

En segundo lugar, es habitual en las series climatológicas el que las variaciones que presentan no se deban, usualmente, únicamente a factores climáticos. En la mayor parte de las ocasiones estas variaciones, saltos abruptos o tendencias en las series, obedecen a factores no climáticos como la introducción de nuevo instrumental, la relocalización de los observatorios, cambios en la exposición de los instrumentos o en las practicas observacionales, modificaciones del entorno del observatorios y otros. Al mismo tiempo, incorporados en las mediciones existen también datos erróneos o aberrantes fruto de causas múltiples (errores de lectura, de transcripción, malfuncionamiento del instrumental, etc...; Aguilar et al., 2003).

Estos factores no climáticos afecta a los registros meteorológicos impidiendo que sean representativos de las variaciones actuales del clima, reduciendo la credibilidad de estas series temporales.

Es indispensable, antes de poder actuar sobre los datos para corregir estas anomalías, recopilar toda la información disponible sobre el observatorio, sus cambios, las modificaciones de su entorno y toda la información posible sobre las causas probables de error o sesgo en los datos, como quien, cuando, donde y como los datos fueron registrados.

Un paso más para la generación de bases de datos climáticos de calidad consiste en el control de calidad (QC) de los datos que van a ser introducidos en esta base de datos. Algunos de estos procedimientos referentes a la Global Daily Climatology Network (GDCN), la European Climate Assessment \& Dataset y la base australiana de temperatura pueden consultarse respectivamente en NCDC (2002), Klein-Tank et al. (2002) y Trewin (1999).

Finalmente, el principal proceso destinado a corregir la influencia de los factores no climáticos en las series de datos meteorológicos es el proceso de detección y corrección 
de inhomogeneidades en los datos. Existen múltiples técnicas que se han desarrollado en las últimas dos décadas para testar la homogeneidad de las series de datos. Las primeras se orientaron a corregir datos en escala anual y mensual, y buena parte de ellas se recogen en Peterson et al., (1998), mientras que en la actualidad existen diversas metodologías orientadas a la detección y corrección de inhomogeneidades en base diaria. Entre ellos se puede citar el Multiple Analysis of Series for Homogenization (MASH; Szentimrey, 1999), el Caussinus-Mestre (Caussinus y Mestre, 2004), HOME (Della-Martay Wanner, 2006) o la aproximación del C3 (Brunet et al, 2007).

El objetivo de este trabajo es ofrecer una metodología para el desarrollo de bases de datos de largo recorrido de temperatura diaria ajustada. Para ello, en las siguientes secciones se detalla el procedimiento seguido para la construcción de la base de datos de Series Diarias Ajustas de Temperatura de España (SDATS) por parte de los autores.

En la primera sección de recogen los procedimientos seguidos para la localización y rescate de datos y metadatos; en la segunda el proceso de control de calidad aplicado a los datos; en la tercera, la metodología empleada para la detección y corrección de las rupturas de homogeneidad; y en la última sección se recoge un análisis del cambio térmico observado en España a partir de SDATS y la metodología seguida para la generación de señales regionales de las variables de temperatura.

\section{Construcción y ajuste de la base de datos}

\subsection{Localización y rescate de datos y metadatos en SDATS}

La primera tarea emprendida en la generación de una base de datos histórica de alta calidad consiste en la identificación de instituciones y entidades susceptibles de disponer de colecciones de registros climáticos. Tradicionalmente los datos meteorológicos han sido registrados y archivados por individuos, instituciones científicas y servicios meteorológicos oficiales desde los inicios de los registros instrumentales, e independientemente de cuales fueran las instituciones a cargo de ello, miles de millones de observaciones fueron registradas y preservadas durante todo el periodo instrumental.

Sin embargo, muchos de estos datos continúan en los formatos originales en que fueron archivados, en copias impresas u otros medios frágiles que no facilitan su inmediato uso por parte de la comunidad científica (papel, microfichas, etc.). En el caso de los registros mas antiguos, su dispersión suele ser mayor y acostumbran a encontrarse en Academias Nacionales de Ciencia y otras instituciones científicas seculares, archivos históricos de los Servicios Meteorológicos Nacionales (SMN) y otros organismos con responsabilidades en datos meteorológicos (servicios hidrológicos, por ejemplo), archivos y bibliotecas de carácter internacional, colonial, nacional o local así como en hemerotecas nacionales y locales. En el caso de datos y metadatos más recientes, la fuente principal la constituyen los archivos centrales o de los centros regionales de los SMN.

La generación de la base de datos SDATS se planteó en el marco del proyecto europeo EMULATE (European and North Atlantic daily to MULtidecadal climATE variability) con el objetivo de desarrollar una nueva base de datos diarios ajustado para el conjunto de España que pudiera ser utilizada para realizar una análisis fiable y robusto de la variabilidad y el cambio en los registros térmicos de largo recorrido.

En la elección de los observatorios para formar parte de SDATS se siguieron diversos criterios: cobertura espacial y temporal; representatividad climática; continuidad de los datos; y calidad potencial de los registros. Por ello, los observatorios seleccionados tenían que remontar sus registros al siglo XIX, o como mínimo cubrir el conjunto del siglo XX, ser 


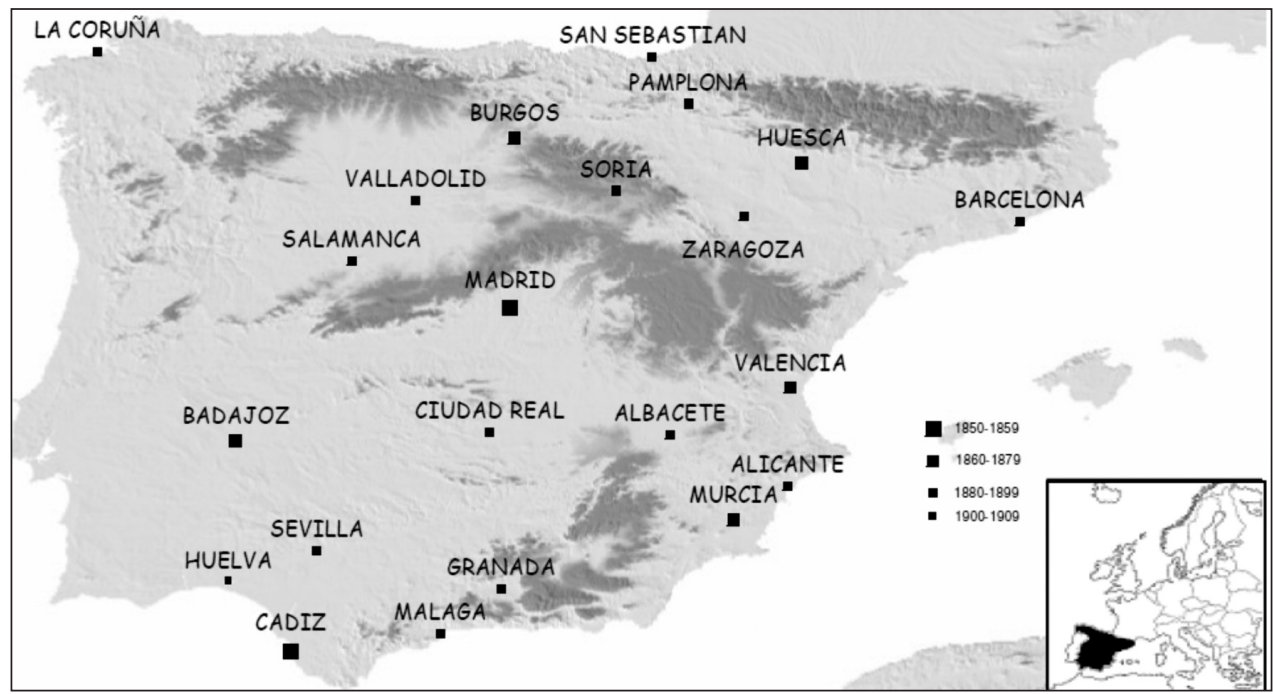

FIGURA 1. Mapa de localización de los observatorios integrados en la base de datos SDATS y extensión de los registros de temperatura.

representativos de la riqueza climática española, tener continuidad pasada y presente en su funcionamiento y estar catalogadas como estaciones de primer orden por el entonces Instituto Nacional de Meteorología (INM), actual Agencia Estatal de Meteorología (AEMet).

De este proceso surgió la selección de los 22 observatorios, las series de los cuales se integran en la SDATS, que aparecen localizados en la Figura 1. En conjunto se localizan 9 observatorios costeros ( 2 en la costa atlántica norte, 5 en la costa mediterránea y 2 en la costa atlántica sur), 8 en la meseta (4 en la meseta norte y 4 en la meseta sur), 3 en el valle del Ebro y 2 en el valle del Guadalquivir. Esta red cubre los principales tipos y subtipos climáticos identificados para el territorio español por Martín-Vide y Olcina (2001).

Un criterio adicional utilizado en la selección de los observatorios fue componer las series con datos registrados en pequeñas y medianas ciudades durante el siglo XIX y primera mitad del XX, mientras que para la segunda mitad del siglo XX se utilizaron principalmente observatorios cercanos ubicados en áreas no urbanas, en su mayoría aeródromos y aeropuertos o en elevaciones sobre la ciudad.

Los datos originales que conforman las series de $\mathrm{T}_{\text {maxima }}$ y $\mathrm{T}_{\text {minima }}$ de estos 22 observatorios se obtuvieron de una amplia variedad de fuentes, aunque el grueso de esos datos (80\%) fue obtenido en formato digital e impreso del INM (48\% y 32\%). El resto fueron recuperado en formato digital (14\%) e impreso $(6 \%)$ de otras fuentes. La Tabla I proporciona información sobre los organismos de los cuales se ha obtenido datos y metadatos y las fuentes documentales utilizadas. La mayor parte del siglo XX esta cubierto principalmente con datos facilitados por el INM, mientras que otras fuentes proporcionan en gran parte los datos pertenecientes al siglo XIX.

Los autores han localizado y recuperado datos antiguos obtenidos de fuentes impresas y han digitalizado cerca del $40 \%$ de total de datos recuperados, un volumen cercano a los 2 millones de observaciones diarias de temperatura. Las observaciones meteorológicas en España se inician oficialmente en 1869 , aunque antes de esta fecha observatorios no oficiales 
funcionaron en muchas ciudades españolas y, como era habitual en la época, los datos se intercambiaban entre observatorios nacionales e internacionales, principalmente mediante retransmisiones telegráficas y se publicaban en boletines diarios o semanales o en resúmenes mensuales o anuales. Igualmente habitual era que los periódicos locales ofrecieran a sus lectores los datos meteorológicos diarios de su ciudad y de otras, tanto a escala nacional como internacional, lo que convierte estas publicaciones en fuentes susceptibles de ser utilizadas para la recuperación de datos diarios. Pero como en otras fuentes indirectas en las que los datos sufren un cierto grado de manipulación, la credibilidad de la fuente es menor que en el caso de fuentes originales.

La Tabla I indica las principales fuentes utilizadas para recuperar los datos de las series integradas en SDATS. El Daily Weather Report (DWR) constituye un boletín diario de datos climáticos del siglo XIX, que recoge información diaria recibida por la Oficina Meteorológica del Reino Unido (UK-MO) retransmitida por telégrafo desde diferentes observatorios meteorológicos europeos. Similar a este encontramos el Boletín Meteorológico Diario (BMD), boletín compilado por el Instituto Central Meteorológico con sede en Madrid (ICM), que recoge datos diarios de observatorios del conjunto del territorio español. Ambas fuentes presenta un alto grado de fiabilidad ya que, aunque se trata de fuentes indirectas, estas fueron manejadas por observadores concienciados tanto en los lugares de emisión como en los de recepción. El principal problema con estos datos reside en los datos ausentes por problemas en la comunicación, aunque se trata de fuentes muy completas en general.

La publicación de Rico Sinobas es un documento que recoge y analiza las observaciones diarias registradas en el Observatorio de Madrid por parte de M. Rico Sinobas, el observador a cargo del Real Observatorio Astronómico de Madrid (ROAM), para el periodo de los años 1853 y 1854 . Aunque se trata de una documentación indirecta, su fiabilidad es elevada al tratarse de transcripciones de las fuentes originales realizadas por el observador, no descartando la posibilidad de algunos errores tipográficos.

Tanto la monografía del ICM (1893) que contiene 30 años (1860-1889) de observaciones diarias de datos climáticos para el Observatorio de Madrid como las del Real Observatorio Astronómico de Madrid (1892, 1894), que recoge los datos entre 1890 y 1893 para el mismo observatorio, son transcripciones de los originales realizadas por la misma institución que toma los datos y su fiabilidad es alta.

De menor fiabilidad son las dos fuentes restantes, las publicaciones de prensa diaria $L a$ Gaceta de Madrid (LG) y El Noticioso (EN), ya que se trata de las fuentes más indirectas utilizadas en esta base de datos. Este último diario resultó ser el menos útil, ya que se constató que al menos el 30\% de los datos en 3 años de observaciones diarias eran no creíbles, principalmente datos repetidos de días anteriores, principalmente los fines de semana y los lunes. Este hecho condujo a los investigadores a desechar esta fuente como valida y los datos correspondientes al EN no se incorporaron a la base de datos.

En contraste, LG resulto ser una fuente más creíble de datos climáticos diarios continuos. Este diario publico las observaciones diarias registradas por la red meteorológica española durante la segunda mitad del siglo XIX, transmitida por el ICM. A pesar de ello, los errores tipográficos en este tipo de fuentes son más posibles que en las otras publicaciones citadas, por lo que todos los datos obtenidos a partir de esta y las otras fuentes deben ser sometidos a un riguroso control de calidad (QC).

De estas y otras fuentes recogidas en la Tabla I, los autores compilaron también metadatos para el conjunto de la red, con el objeto de facilitar la detección de rupturas de homogeneidad en los registros. Aunque lejos de ser exhaustiva, esta metadata contenía información acerca de identificadores de los observatorios, localización geográfica, tipo 
Tabla I

\begin{tabular}{|c|c|}
\hline Organismos & $\begin{array}{l}\text { Detalles de los datos y metadatos recuperados en las fuentes } \\
\text { documentales }\end{array}$ \\
\hline INM, SDC, Madrid, & $\begin{array}{l}\text { Datos: } 21 \text { series cubriendo el siglo XX en copias de papel y digital, con } \\
\text { control de calidad de errores aberrantes realizado por SDC en una fracción } \\
\text { de los datos digitalizados. }\end{array}$ \\
\hline CMTM, Murcia & $\begin{array}{l}\text { Datos: Un registro de temperatura (Murcia), 1863-1950, QC de errores } \\
\text { aberrantes realizado por el servicio territorial del INM en Murcia. }\end{array}$ \\
\hline UE: proyecto IMPROVE & $\begin{array}{l}\text { Datos: un registro digitalizado (Cádiz), 1850-1996, QC de errores aberrantes } \\
\text { (Camuffo and Jones, 2002). Metadatos parciales del observatorio de Cádiz en } \\
\text { Barriendos et al. (2002) }\end{array}$ \\
\hline ROASF, Cadiz & $\begin{array}{l}\text { Datos: actualización de Cadiz, 1997-2005, QC de errores aberrantes del } \\
\text { ROASF. Metadatos: Metadatos parciales del observatorio de Cádiz en } \\
\text { González (1992) }\end{array}$ \\
\hline $\begin{array}{l}\text { Pavel Groisman, NCDC, Asheville, NC, } \\
\text { USA }\end{array}$ & $\begin{array}{l}\text { Datos: Dos registros de digitalizados: Valencia 1937-1999 y Salamanca } \\
\text { 1945-1999. NCDC (2002). }\end{array}$ \\
\hline EC: MB_ADVICE & Datos: Un registro digitalizado (Barcelona, 1885-1900). \\
\hline $\begin{array}{l}\text { Archivo y Biblioteca Nacional de la } \\
\text { Oficina Meteorológica del Reino Unido } \\
\text { (UK-MO), Bracknell, Reino Unido }\end{array}$ & $\begin{array}{l}\text { Datos: Daily Weather Reports (DWR): Un registro (La Coruña, 1882-1900), } \\
\text { Boletín Meteorológico Diario (BMD): } 12 \text { registros, 1893-1900 }\end{array}$ \\
\hline Biblioteca y Archivo del INM, Madrid & $\begin{array}{l}\text { Datos: Madrid, 1853-1854, en Rico Sinobas (1857). Madrid, 1860-1889, en } \\
\text { ICM (1893). Madrid, 1890-1892, en ROAM (1892,1894). } \\
\text { Metadatos para el conjunto de la red disponible en: Observatorio de Madrid } \\
\text { (1866-1880, 1882, 1884, 1886-1887, 1889, 1891-1892, 1895-1896, 1899- } \\
\text { 1900): Resumen de las observaciones meteorológicas efectuadas en la } \\
\text { Peninsula y algunas de sus islas adyacentes. Diversas editoriales: Madrid; ICM } \\
\text { (Instituto Central Meteorológico) (1907-1949): Resumen de las observaciones } \\
\text { meteorológicas efectuadas en la Península y algunas de sus islas adyacentes. } \\
\text { Est. Tipo-litográfico de I. Barredo: Madrid, y Almarza et al.(1996). }\end{array}$ \\
\hline Real Academia de Medicina, Madrid & $\begin{array}{l}\text { Datos: Madrid, } 1855 \text {, en RSM: Observaciones meteorológicas de Madrid: } \\
\text { 1800-1857, (23 v.) v. } 21,(12-8-M-4-23)\end{array}$ \\
\hline Hemeroteca Municipal, Tarragona & Datos: Madrid, 1856-1859, en el diario de prensa La Gaceta (LG). \\
\hline $\begin{array}{l}\text { Biblioteca de la Sociedad Económica de } \\
\text { Amigos del País, Badajoz }\end{array}$ & Metadatos parciales para Badajoz de Sánchez Pascua (1985) \\
\hline $\begin{array}{l}\text { CMTG (Centro Meteorológico } \\
\text { Territorial de Galicia), Archivo } \\
\text { Climatologico, La Coruña }\end{array}$ & $\begin{array}{l}\text { Metadatos disponibles para La Coruña en Rios Pardo L. 2000. El observatorio } \\
\text { meteorológico (1). CMTG, informe interno: La Coruña y en «La Ilustración } \\
\text { Gallega y Asturiana» prensa diaria, n 16, } 8 \text { de junio, } 1881 \text {. }\end{array}$ \\
\hline
\end{tabular}

Fuente de los datos y metadatos integrados en SDATS y detalle de la información suministrada. Acrónimos: INM: Instituto Nacional de Meteorología; DWR: Daily Weather Reports; BMD: Boletín Meteorológico Diario; MB-ADVICE: Mariano Barriendos y projecto europeo ADVICE (Annual to Decadal Variability In Climate in Europe); ICM/ROAM: Instituto Central Meteorologico/Real Observatorio Astronómico de Madrid; RSM: Ricos Sinobas, Manuscrito; LG: La Gaceta de Madrid; PG: Pavel Groisman; CMTM: Centro Meteorológico Territorial en Murcia del INM; IMPROVE: Proyecto europeo Improved Understanding of Past Climatic Variability from Early European Instrumental Data; ROASF: Real Observatorio de la Armada en San Fernando (Cádiz, España). 
y subtipos climáticos, datos sobre las relocalizaciones de los observatorios en diferentes ubicaciones, unidades de medida, datos ausentes y fuente de los datos. Adicionalmente, también se incluyó información parcial sobre los alrededores de los observatorios a meso y micro escala cuando fue posible.

\subsection{Control de calidad de los datos originales}

El análisis exhaustivo de la calidad de los datos es un paso esencial antes de realizar cualquier prueba de homogeneidad o cualquier análisis de la variabilidad del cambio climático a largo plazo, especialmente con datos a escala diaria. Un QC bien definido y ejecutado previene de errores potenciales que pueden comprometer los ajustes realizados por cualquier prueba de homogeneidad o modificar los análisis realizados sobre los datos, como la evaluación del comportamiento extremo del clima.

El QC no consiste únicamente en la identificación de posibles errores. Los datos que no superan las diferentes pruebas de QC deben ser etiquetados como sospechosos y debe consultarse en la fuente original si se han introducido o importado correctamente, lo que permite recuperar una fracción importante de los datos sospechosos de ser erróneos, ya sea validándolos o sustituyéndolos por los valores correctos. Únicamente aquellos datos sospechosos que no puedan validarse o recuperarse son eliminados de la base de datos.

El conjunto de pruebas de QC realizadas sobre los datos originales de las 22 series originales de $\mathrm{T}_{\text {maxima }}$ y $\mathrm{T}_{\text {minima }}$ incluía pruebas diseñadas para identificar errores introducidos durante el proceso de digitalización, manipulación, formateo, transmisión o archivo de los datos. Como en la mayoría de los casos los observatorios fueron trasladados de su ubicación inicial al menos una vez, el QC se realizó por separado para cada uno de estos tramos, con objeto de usar los umbrales más realistas para cada uno de ellos. Este conjunto de pruebas recomendadas (Aguilar et al., 2003; Brunet et al., 2008) fueron las siguientes:

1) Errores aberrantes

i. Valores aberrantes $\left(\mathrm{T}>50^{\circ} \mathrm{C}\right.$ o $<-50^{\circ} \mathrm{C}$, Precipitación negativa, ...)

ii. Consistencia con el calendario. ( $\mathrm{n}^{\mathrm{o}}$ días por año y $\mathrm{n}^{\circ}$ de días por mes)

iii. Comparación de medias mensuales entre las calculadas a partir de los datos diarios digitalizados y los contenidos e fuentes originales accesibles.

2) Pruebas de tolerancia

i. 4 o mas valores idénticos sucesivos

ii. valores a mas de \pm 4 desviaciones típicas

3) Consistencia interna $\left(T_{\text {máxima }}<T_{\text {mínima }}\right)$

4) Coherencia temporal (valores con diferencias superiores a $25^{\circ} \mathrm{C}$ entre observaciones consecutivas)

5) Coherencia espacial

i. Valores que exceden el umbral de $\pm 4 \sigma$ entre la serie candidata y su grupo de series de referencia

ii. Comparación visual con series de observatorios cercanos.

Del total de datos controlados de calidad (1.981.192), un $0.58 \%$ (11.505) fueron etiquetados como sospechosos. De ellos, mas del $70 \%$ fueron validados o recuperados tras consulta a las fuentes originales o, en los casos en los que este procedimiento no era posible, el juicio experto de los investigadores considerando los valores adyacentes en la misma serie de datos, otras variables climáticas del mismo observatorio (precipitación, presión) y la comparación con observaciones simultaneas de observatorios cercanos con 
buena correlación, decidió su permanencia o exclusión de la base de datos. En total, el $30 \%$ (3.415) de los datos sospechosos fueron desechados y considerados en la base de datos como datos ausentes.

La mayoría de los errores encontrados estaban relacionados con valores mal introducidos durante el proceso de digitalización.

\subsection{Detección y corrección de rupturas de homogeneidad}

Tras la validación, reemplazo o eliminación de los datos sospechosos en el QC, el siguiente paso adoptado para la generación de la base de datos de alta calidad SDATS fue detectar y corregir problemas en la homogeneidad de las series. Para ello se utilizaron 3 técnicas diferenciadas.

En primer lugar se empleó una aproximación empírica para minimizar el denominado efecto «screen», sesgo producido por las diferencias en los abrigos meteorológicos utilizados en los observatorios a lo largo de la historia de la estación y que afecta usualmente a la temperatura del siglo XIX y principios del siglo XX.

En segundo lugar, se utilizó una aplicación del Standard Normal Homogeneity Test (SNHT) desarrollado por Alexandersson y Moberg (1997) para la detección de puntos de ruptura de homogeneidad, establecer los patrones de corrección y estimar los ajustes mensuales requeridos.

En tercer lugar, se adopto un procedimiento de interpolación basado en el esquema desarrollado por Vincent (2002) para adaptar los factores de ajuste mensual a los datos en escala diaria.

\subsubsection{Minimización del efecto «screen» en la red SDATS}

El efecto que los cambios en el tipo de abrigo meteorológico tiene sobre los registros térmicos fue documentado por Parker (1994), indicando que las lecturas de temperatura efectuadas sobre diferentes tipos de garitas presentaban un sesgo cuyo signo depende de la latitud, el momento el día y año en que se efectúa la medición y la variable de temperatura extrema (máxima o mínima) afectada. En el clima mediterráneo, Nicholls (1996) advirtió que los registros antiguos de temperatura presentaban fuertes sesgos cálidos en la $\mathrm{T}_{\text {máxima }}$ comparados con los actuales, mientras que la $\mathrm{T}_{\text {mínima }}$ mostraba un sesgo menor y de signo contrario.

La red meteorológica española decimonónica usaba de forma preferente garitas abiertas para albergar los termómetros (tipo Montsouris y, en menor grado, Glaisher), que son mas sensibles que las actuales garitas Stevensson a los cambios radiactivos del entorno inmediato. Esta diferencia en el tipo de garita utilizada durante el siglo XIX y primeras décadas del siglo XX ha provocado un sesgo en el conjunto de registros de temperatura históricos que afecta a todos ellos en mayor o menor grado, y que difícilmente puede detectarse y corregirse con pruebas relativas de homogeneidad.

Por ello, en el marco del proyecto nacional SCREEN (REN2002-0091/CLI) se han realizado medidas pareadas de temperatura entre reconstrucciones de garitas tipo Montsouris (según descripciones de Angot, 1903) y las actuales Stevenson en los jardines meteorológicos de La Coruña y Murcia, representativos del clima oceánico peninsular y mediterráneo árido, con alta y baja cobertura nubosa respectivamente. El objetivo de este proyecto es estimar la magnitud y fluctuaciones del sesgo cálido (frío) sobre la temperatura máxima (mínima) diaria con objeto de desarrollar procedimientos analíticos de minimización de este efecto en los registros españoles históricos de temperatura. 
Una primera aproximación a la minimización del sesgo se recoge en Brunet et al. (2006) a partir de un año de observaciones, mientras que la corrección efectuada a SDATS a partir de 2 años de observaciones pareadas, se detalla en Brunet et al. (2007, 2008). Esta aproximación se basa en la alta correlación y relación lineal existente entre las observaciones de la temperatura efectuadas en ambas garitas, y consiste en dos modelos de regresión lineal, uno para cada localización usando la $\mathrm{T}_{\text {máxima }}$ Montsouris para predecir los valores de $\mathrm{T}_{\text {máxima }}$ en el abrigo actual Stevensson. Ambos modelos explican el $99 \%$ de la varianza de la $\mathrm{T}_{\text {máxima }}$ en Murcia y el $98.6 \%$ en La Coruña y se expresan como:

$$
\begin{gathered}
\mathrm{Tc}=-0.508+(0.975 \mathrm{Tr}) \text { para Murcia y } \\
\mathrm{Tc}=0.059+(0.949 \mathrm{Tr}) \text { para La Coruña, }
\end{gathered}
$$

donde Tr es la temperatura original medida en la garita Montsouris y Tc la temperatura corregida como si estuviera medida en garita Stevensson.

Esta corrección se aplicó a los datos originales de temperatura máxima diaria hasta la fecha en que los metadatos disponibles indica la substitución de la garita de tipo abierto a la garita tipo Stevenson (Tabla II). La excepción corresponde al observatorio de Málaga, en el que por un periodo indeterminado de tiempo los termómetros fueron expuestos, entre las ultimas décadas del siglo XIX y principios del siglo XX, dentro de un abrigo de persianas de $2 \mathrm{~m} \times$ de $3 \mathrm{~m} \times 2 \mathrm{~m}$ con una puerta abierta al norte, que tuvo que tener una influencia diferente en las lecturas que el sesgo inducido por los abrigos abiertos.

El sesgo en la $\mathrm{T}_{\text {mínima }}$ inducido por la exposición abierta es de menor magnitud y no muestra un ciclo anual definido a lo largo del año como sucede con la temperatura máxima. La aproximación por modelo de regresión lineal no era aplicable en este caso, por lo que se optó por utilizar las diferencias en las medianas mensuales estimadas a partir de las observaciones pareadas de la temperatura minima diaria de los dos años empleados en el estudio (Tabla III) sobre los datos originales de $\mathrm{T}_{\text {mínima }}$ para los periodos definidos en la Tabla II.

Aunque esta metodología para la minimización del sesgo «screen» es la última presentada y validada por los autores, se están desarrollando nuevas aproximaciones con un mayor volumen de datos pareados, que se recogerán en Brunet et al. (2010).

Tabla II

FECHAS DE INTRODUCCIÓN DE LA GARITA TIPO STEVENSON EN LA RED PERIODO ANTERIOR A ESTA FECHA. EN NEGRITA (CURSIVA) LAS ESTACIONES AJUSTADAS MEDIANTE LA CORRECCIÓN PARA MURCIA (LA CORUÑA)

\begin{tabular}{|c|c|c|c|c|c|c|c|}
\hline Albacete & $4 / 1915$ & Alicante & $1 / 1909$ & Badajoz & $1 / 1909$ & Barcelona & $1 / 1901$ \\
\hline Burgos & $1 / 1905$ & Cadiz & $1 / 1875$ & $\begin{array}{c}\text { Ciudad } \\
\text { Real }\end{array}$ & $1 / 1908$ & Granada & $1 / 1909$ \\
\hline Huelva & $1 / 1909$ & Huesca & $6 / 1912$ & La Coruña & $4 / 1912$ & Madrid & $1 / 1894$ \\
\hline Murcia & $1 / 1913$ & Pamplona & $1 / 1916$ & Salamanca & $1 / 1909$ & S. Sebastian & $1 / 1901$ \\
\hline Sevilla & $5 / 1912$ & Soria & $1 / 1914$ & Valencia & $1 / 1901$ & Valladolid & $10 / 1912$ \\
\hline Zaragoza & $4 / 1913$ & \multicolumn{7}{|c|}{} \\
\hline
\end{tabular}




\subsubsection{La aplicación del SNHT a los datos mensuales}

Tras la aplicación de los ajustes del sesgo provocado por el efecto «screen» en los datos originales, se procede a la segunda etapa del proceso de detección y ajuste de la homoge-

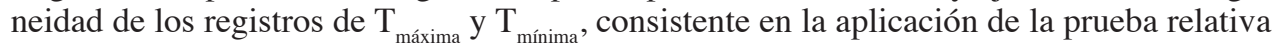
SNHT de Alexandersson y Moberg (1997) siguiendo el esquema de detección y corrección de inhomogeneidades en series mensuales de temperatura descrito por Aguilar et al., (2002). Este esquema indica un primer paso en el cual se deben seleccionar un conjunto de 8-9 observatorios o estaciones de referencia para cada estación candidata a la cual se aplica la prueba, un segundo paso que consiste en la detección de rupturas de homogeneidad, y por último la definición de un patrón de corrección aplicable a los promedios mensuales de la temperatura máxima y minima.

Tabla III

AJUSTES MENSUALES (EN ${ }^{\circ} C$, CON EL INTERVALO DE CONFIANZA AL 95\% ENTRE PARÉNTESIS) ESTIMADOS DE LA SERIE DIFERENCIA ENTRE LA TEMPERATURA MINIMA DIARIA REGISTRADA EN LA GARITA MONTSOURIS $Y$ EN LA STEVENSON EN LOS JARDINES METEOROLÓGICOS DE LA CORUÑA $Y$ MURCIA PARA LA MINIMIZACIÓN DEL SESGO «SCREEN»EN LOS ABRIGOS METEOROLÓGICOS PRE-STEVENSSON.

\begin{tabular}{|c|c|c|}
\hline Meses & La Coruña & Murcia \\
\hline Enero & $0.18(0.14 / 0.22)$ & $0.27(0.21 / 0.33)$ \\
\hline Febrero & $0.16(0.10 / 0.21)$ & $0.19(0.14 / 0.24)$ \\
\hline Marzo & $0.17(0.13 / 0.21)$ & $0.13(0.08 / 0.18)$ \\
\hline Abril & $0.14(0.09 / 0.19)$ & $0.16(0.10 / 0.22)$ \\
\hline Mayo & $0.14(0.10 / 0.17)$ & $0.16(0.10 / 0.22)$ \\
\hline Junio & $0.21(0.17 / 0.24)$ & $0.21(0.16 / 0.26)$ \\
\hline Julio & $0.17(0.14 / 0.20)$ & $0.13(0.09 / 0.17)$ \\
\hline Agosto & $0.26(0.22 / 0.30)$ & $0.19(0.14 / 0.24)$ \\
\hline Septiembre & $0.24(0.20 / 0.29)$ & $0.11(0.06 / 0.17)$ \\
\hline Octubre & $0.20(0.16 / 0.23)$ & $0.27(0.22 / 0.32)$ \\
\hline Noviembre & $0.19(0.11 / 0.27)$ & $0.21(0.15 / 0.26)$ \\
\hline Diciembre & $0.19(0.12 / 0.26)$ & $0.28(0.23 / 0.33)$ \\
\hline
\end{tabular}

La elección de las estaciones de referencia para cada estación candidata se basa en 3 criterios complementarios: un coeficiente de correlación elevado; proximidad geográfica y/o afinidad climática; y disponibilidad del suficiente numero de series de referencia para cualquier ventana temporal de la serie candidata.

Los coeficientes de correlación empleados se han calculado respecto las series de primera diferencia, para prevenir distorsiones debido a las potenciales inhomogeneidades. Debido a que el número de series disponibles durante la segunda mitad del siglo XIX se reduce progresivamente, el conjunto de estaciones de referencia se ha reducido para este periodo dependiendo de la estación candidata analizada, considerándose necesario un mínimo de 3 estaciones de referencia para poder aplicar el SNHT. Para aquellos tramos 
en que las series no cumplen estos requisitos (Tabla IV), se les han aplicado los factores de corrección obtenidos por el SNHT para el resto de tramos de la serie.

La mayoría de las series de referencia muestran correlaciones superiores a 0.80 con la estación candidata, aunque en algunos casos se han añadido estaciones con correlaciones entre 0.70-0.75 para poder garantizar la disponibilidad de series de referencia en determinados tramos, como el siglo XIX o el periodo en torno a la Guerra Civil.

Tras la elección de las series de referencia, la etapa inicial de la aplicación del esquema de detección y corrección del SNHT, denominada aplicación «no guiada», se inició con la ejecución del SNHT sobre las series de promedios anuales y estacionales de $\mathrm{T}_{\text {máxima }}, \mathrm{T}_{\text {mínima }}$ $\mathrm{y}_{\text {media }}$, con objeto de detectar potenciales inhomogeneidades en las tres variables y en las dos escalas temporales.

El patrón de corrección para cada estación candidata se construye a partir de la inspección de los puntos de ruptura de homogeneidad detectados por la aplicación no guiada del SNHT, el análisis de las Q-series (la diferencia entre la serie candidata y el promedio ponderado de las series de referencia) derivadas de la aplicación del SNHT y de la información disponible a partir de los metadatos, con objeto de asociar cada punto de ruptura de homogeneidad definido estadísticamente con un factor físico que pueda explicarlo.

\section{Tabla IV \\ TRAMOS DE LAS ESTACIONES QUE NO CUMPLEN EL REQUISITO DE UN \\ MÍNIMO DE 3 ESTACIONES DE REFERENCIA PARA PODER APLICAR EL SNHT Y EN LOS CUALES NO SE HA APLICADO EL TEST}

\begin{tabular}{|c|c|c|c|}
\hline Badajoz & $1864-1875$ & Burgos & $1870-1883$ \\
\hline Cadiz & $1850-1862$ & Huesca & $1861-1882$ \\
\hline La Coruña & $1882-1885$ & Madrid & $1853-1862$ \\
\hline Valencia & $1864-1887$ & & \\
\hline
\end{tabular}

De un total de 108 potenciales puntos de ruptura de homogeneidad, 61 han sido validados en base anual. En los casos en que no se disponía de metadatos estos puntos de cambio en las series se han validado únicamente si estaban presentes a escala anual y en al menos dos series estacionales, tanto para la $\mathrm{T}_{\text {media }}$ como para, al menos, una de las dos

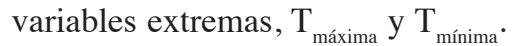

La Tabla $\mathrm{V}$ muestra las fechas de las rupturas de homogeneidad (abruptas o graduales) y sus causas para el conjunto de las 22 estaciones integrantes de SDATS. De las 61 ruptura detectadas, 56 están relacionadas con saltos abruptos en la serie (años individuales en la Tabla V) y únicamente 5 con tendencias graduales (periodos en la Tabla V). Estas tendencias graduales identificadas en las series de $\mathrm{T}_{\text {mínima }}$ se relacionan probablemente con efecto urbano, y fueron validadas tras consultar en los metadatos disponibles acerca del crecimiento urbano (demográfico) durante el periodo afectado.

La principal fuente de inhomogeneidad detectado en las series españolas de SDATS corresponde a los cambios en la localización del observatorio, que causa el $56 \%$ de las inhomogeneidades detectadas. Le siguen las tendencias graduales ejercidas por el desarrollo urbano (9\%), y un $3 \%$ corresponde a cambios en la fuente de los datos. La causa del $34 \%$ de las inhomogeneidades no pudo determinase con la información disponible en ese momento.

El número de inhomogeneidades medio detectado es de 2.7 por estación, correspondiendo 2 puntos de cambio a casi la mitad de la red de observatorios, mientras que tan solo 2 estaciones presentaban con un número de inhomogeneidades superior a 4 . 


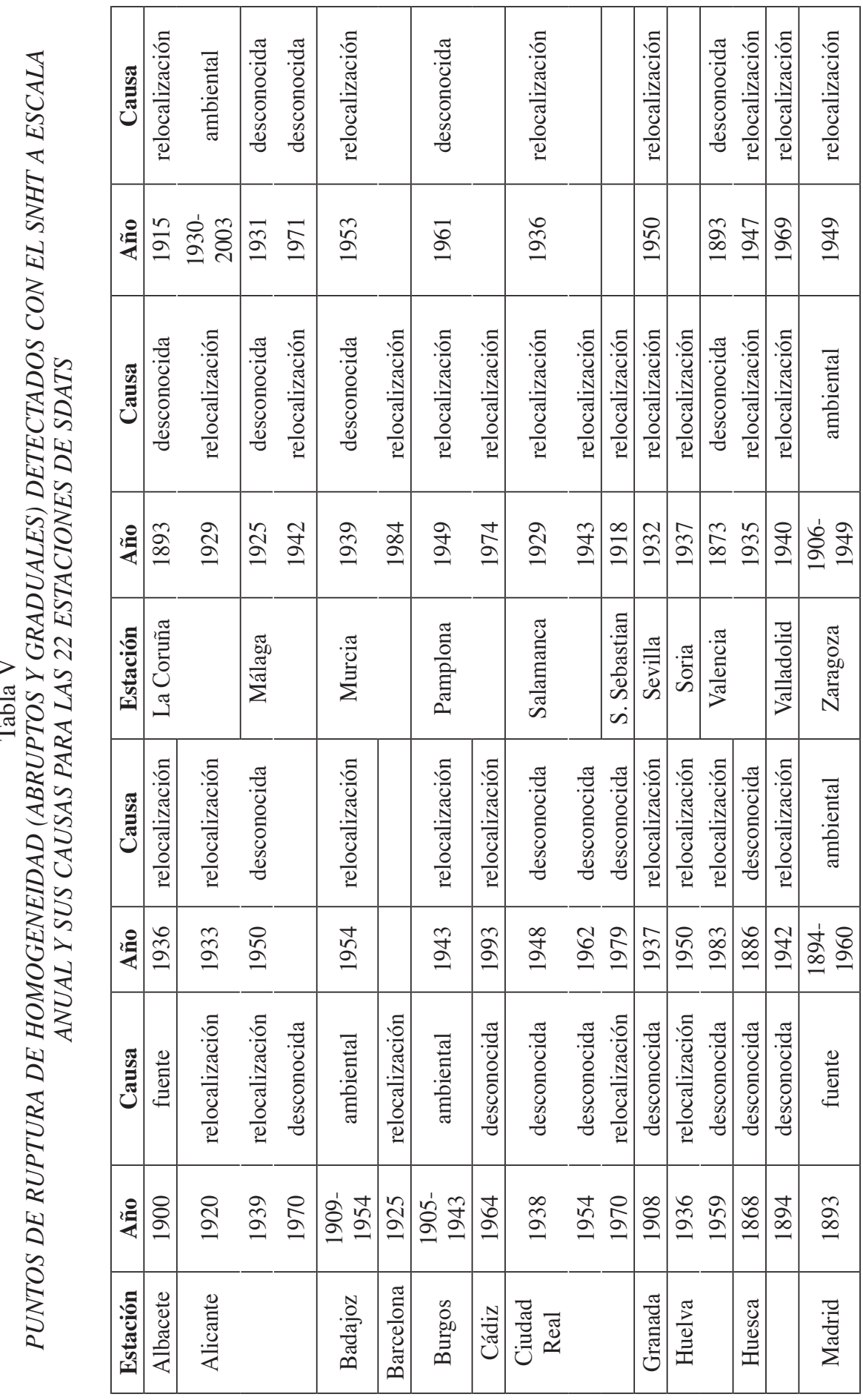


El patrón de corrección identificado para cada estación a escala anual y estacional reflejado en la Tabla $\mathrm{V}$ se aplicó a los 12 series mensuales de $\mathrm{T}_{\text {máxima }} \mathrm{y}_{\text {mínima }}$ de cada estación ajustada de «screen», con objeto de calcular los factores de ajuste mensual estimados por el SNHT.

\subsubsection{La interpolación de los factores mensuales a la escala diaria}

De las diversas aproximaciones metodológicas para el ajuste de los datos diarios a partir de los coeficientes mensuales, los autores seleccionaron el esquema desarrollado por Vincent (2002) y empleado también por Feng et al. (2004) en la homogenización de los datos meteorológicos diarios de China. Esta aproximación proporciona el mejor procedimiento de interpolación, preservando el promedio mensual y no introduciendo discontinuidades artificiales entre el final de un mes y el inicio del siguiente.

La metodología consiste en derivar los ajustes diarios de los factores de corrección calculados en base mensual mediante una interpolación lineal entre unos valores «objetivo» situados en el punto medio de cada mes, escogidos de manera que el promedio de los ajustes diarios sobre un mes determinado sea igual al factor de corrección mensual. Los valores «objetivo» están relacionados con los ajustes mensuales mediante la matriz:

$$
\mathbf{T}=\mathbf{A}^{-1} \mathbf{M}
$$

donde A es una matriz tridiagonal 12 x 12, M es un vector 12 x 1 con los factores de corrección mensual y $\mathbf{T}$ es un vector resultante 12 x 1 con los valores «objetivo». Los valores «objetivo» se asignan al día central de cada mes y finalmente se interpola linealmente para ajustar el dato diario.

Los ajustes que el proceso completo de homogenización ha efectuado sobre las series pueden resumirse en la Figura 2, donde se recogen los promedios de las diferencias a escala anual entre los datos ajustados y los datos originales de la $\mathrm{T}_{\text {máxima }}$ y $\mathrm{T}_{\text {mínima }}$ diaria. Los valores han sido suavizados con un filtro gausiano de 13 años.

Puede observarse el efecto de la minimización del sesgo «screen», especialmente evidente en los valores anteriores a 1910 de la $\mathrm{T}_{\text {máxima }}$, y en menor grado en la $\mathrm{T}_{\text {mínima }}$. El resto de ajustes efectuados sobre las series durante el proceso de homogenización tiende a presentar un promedio mucho menor cercano a 0 en la $\mathrm{T}_{\text {máxima }}$ y en torno a $-0.5^{\circ} \mathrm{C}$ en la $\mathrm{T}_{\text {mínima }}$

\section{La creación de las series regionales de temperatura y el análisis de tendencias observadas}

\subsection{Técnicas utilizadas para la generación de las series regionales de SDATS}

La construcción de series regionales permite dar una señal sintética representativa del conjunto o parte del territorio analizado. Para ello se han calculado series promedio a partir de las anomalías de cada serie respecto su periodo base 1961-1990, siguiendo la metodología de Jones y Hulme (1996). Con objeto de ajustar el sesgo en la varianza de las series regionales asociado al cambiante numero de estaciones que contribuye a la señal en diferentes subperiodos, se adaptó y aplicó la aproximación desarrollada por Osborn et al. (1997), de acuerdo con la expresión:

$$
Y(t)=X(t) \sqrt{\frac{n^{\prime}(t)}{n^{\prime}(n=N)}}
$$



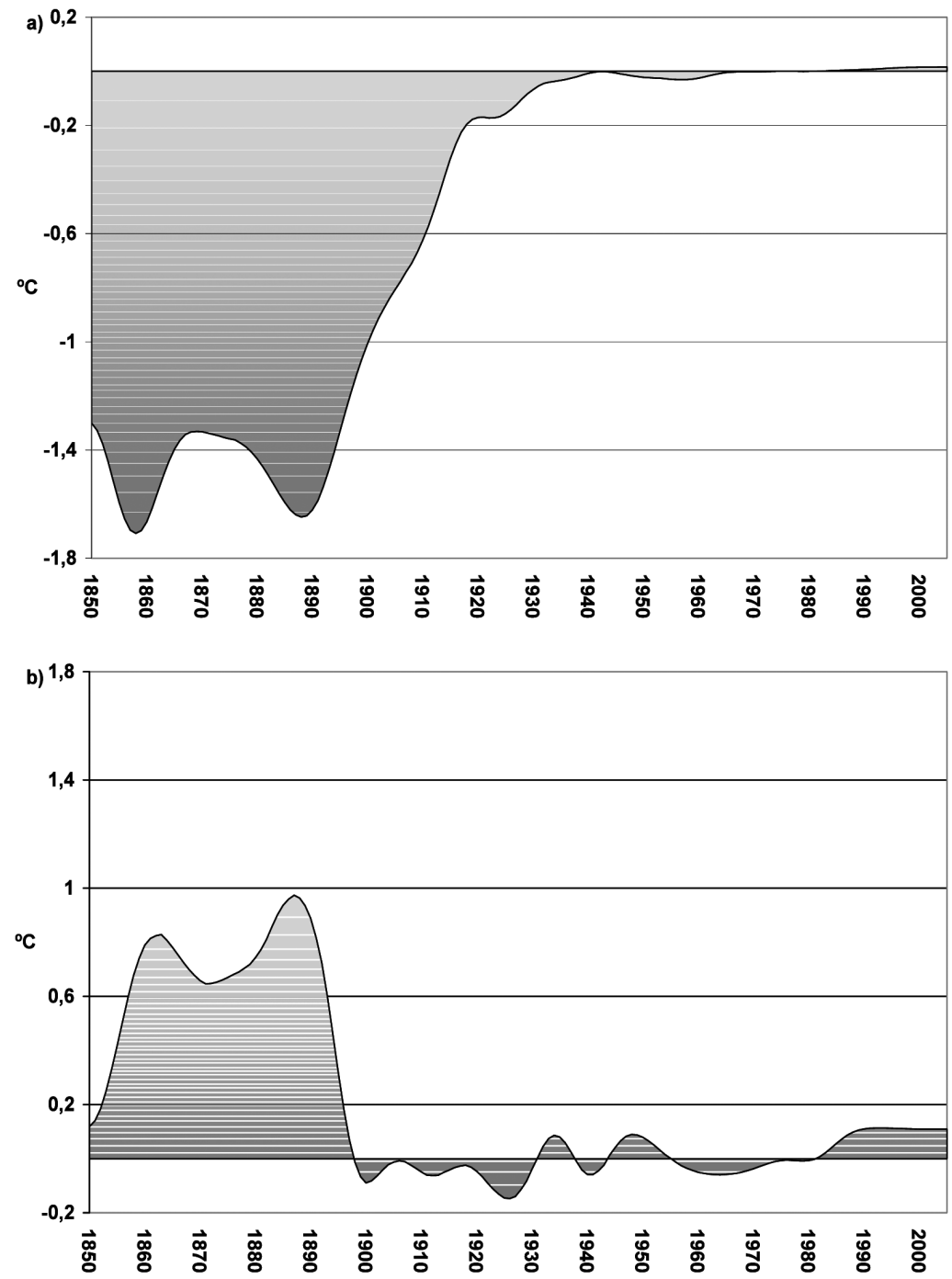

FIgURA 2. Diferencia promedio entre los valores anuales ajustados y los valores anuales originales de a) temperatura máxima diaria y b) temperatura mínima diaria. Curva suavizada mediante un filtro gausiano de 13 términos.

Donde $Y(t)$ es la serie temporal con varianza independiente del número de estaciones, $X(t)$ es la serie regional original, $N$ es el número máximo de estaciones (22 en este caso) y $n$ ’ sigue la expresión:

$$
n^{\prime}=\frac{n(t)}{1+(n(t)-1) \bar{r}}
$$


Donde $n$ es el número de registros y $\bar{r}$ es el promedio de las correlaciones entre todas las parejas de series temporales.

La cuantificación del cambio observado en las variables térmicas se ha calculado mediante un ajuste lineal aplicado a las series anuales y estacionales de $\mathrm{T}_{\text {media }}, \mathrm{T}_{\text {máxima }}$ y $\mathrm{T}_{\text {mínima }}$ mediante una adaptación del estimador de pendiente de Sen (1968) similar a la realizada por Zhang et al (2000). El procedimiento esta basado en un test no paramétrico de MannKendall para detectar la tendencia y el estimador no paramétrico de Sen para calcular la magnitud de la tendencia.

La figura 3 muestra la evolución a escala anual de las tres variables térmicas analizadas a partir de la base SDATS.

\subsection{Análisis de tendencias en SDATS}

En la figura 3 a) puede observarse que la evolución de la temperatura media anual en España no se ha producido de forma gradual y progresiva, distinguiendose diferentes periodos de calentamiento y enfriamiento, de entre los que sobresale el sostenido y vigoroso incremento experimentado por la temperatura española desde inicios de 1970 hasta la actualidad. El anterior periodo de incremento, más moderado (Tabla VI), corresponde al periodo inicial, anterior a 1950, mientras que entre 1950 y 1972 se produce un decremento no significativo en los valores de temperatura.

Similar evolución se detecta en las temperaturas extremas del día (figuras 3 b) y c)), con una mayor contribución al calentamiento en España de los fuertes incrementos en la $\mathrm{T}_{\text {máxima }}$ diaria que de los mas moderados en la $\mathrm{T}_{\text {mínima }}$ diaria (TablaVI), lo que indica que las temperaturas diurnas han tendido a incrementarse mas rápidamente que las nocturnas para el conjunto del periodo. Esta disminución de la amplitud térmica diaria se establece por Alexander et al. (2006) para el $40 \%$ de la tierras emergidas y se corrobora en diversos trabajos que analiza parte del territorio español, como los de Abaurrea et al. (2001) en el Valle del Ebro con 21 estaciones, Brunet et al. (2001) en el noreste español con 23 registros, Galán et al. (2001) en la meseta sur con 24 estaciones, Horcas et al. (2001) en la cuenca del Segura con 23 estaciones y Morales et al. (2005) en la meseta norte con 38 registros de $\mathrm{T}_{\text {máxima }}$ y $\mathrm{T}_{\text {mínima: }}$.

Para el conjunto del periodo analizado, las estaciones con mayor contribución al calentamiento son, en primer lugar, el invierno, con la característica distintiva de ser la única estación del año en que no se observa una disminución de la temperatura durante el periodo 1950-1972, y por lo tanto con un incremento térmico más regular. En segundo lugar, el otoño. Estas dos estaciones son también las que más contribuyen al calentamiento en las

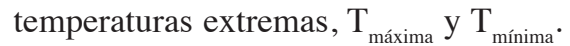

Sin embargo durante la última fase de calentamiento mas vigoroso y significativo, desde inicios de los años 1970's, se detectan diferencias respecto lo descrito para el conjunto del periodo. Aunque se confirma la mayor contribución de la $\mathrm{T}_{\text {máxima }}$ respecto la $\mathrm{T}_{\text {mínima }}$ también para el periodo 1973-2005, las estaciones que mas contribuye al calentamiento de las tres variables térmicas son la primavera y el verano, con tendencias mucho menores en invierno y otoño, siendo únicamente significativas para el otoño de la $\mathrm{T}_{\text {mínima }}$. Este comportamiento rompe la tendencia secular hacia una disminución de la amplitud anual, produciéndose un progresivo distanciamiento entre el rápido calentamiento de la primavera y el verano y el menor ritmo de las otras dos estaciones, similar a lo detectado a escala global por Vose et al. (2005).

Al margen del significativo calentamiento que reflejan las tres variables de la temperatura diaria, en el examen de la evolución térmica española destacan también 2 fases frías 
a) 2

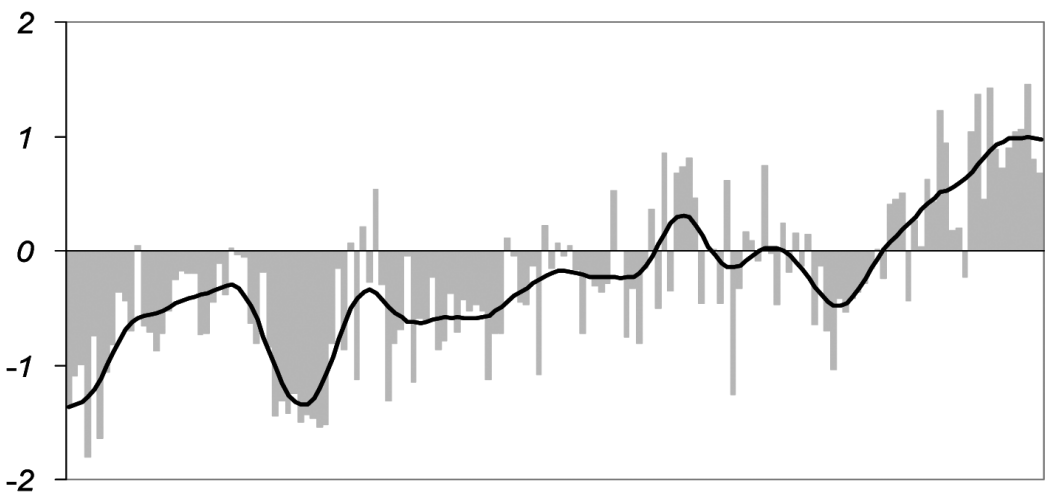

b)

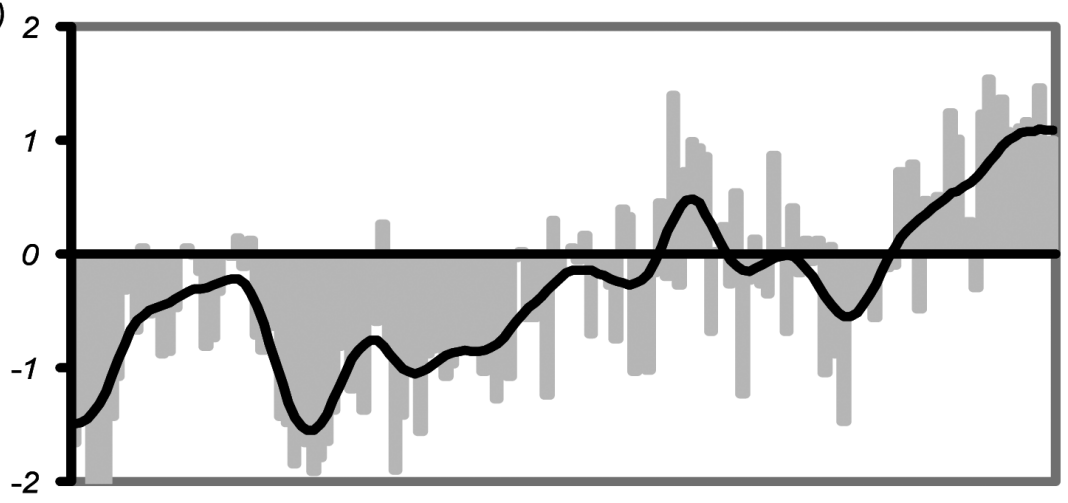

c)

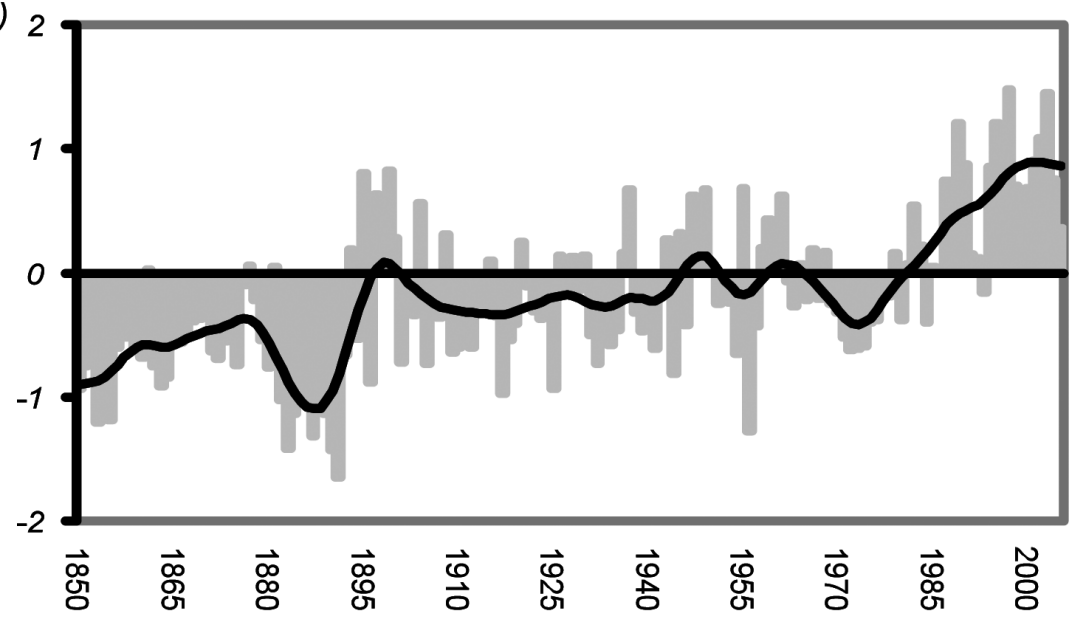

FIGURA 3. Evolución anual para el periodo 1850-2005 de las series regionales españolas de la temperatura media diaria $a$ ), la temperatura máxima diaria $b$ ) y la temperatura mínima diaria c) expresadas como anomalías respecto el periodo 1961-1990 (en ${ }^{\circ} \mathrm{C}$ ). La línea negra corresponde a un filtro gausiano de 13 años. 
bien definidas. La más reciente, también observada a escala global y hemisférica (Jones y Moberg, 2003), entre 1969 y 1978 presenta anomalías de $-0.47^{\circ} \mathrm{C}$, respecto el periodo 1961-1990, a escala anual, con una mayor contribución del otoño $\left(-0.63{ }^{\circ} \mathrm{C}\right)$, la primavera $\left(-0.58^{\circ} \mathrm{C}\right)$ y el verano $\left(-0.53{ }^{\circ} \mathrm{C}\right)$, y mucho menor del invierno $\left(-0.19^{\circ} \mathrm{C}\right)$. La anterior fase fría, localizada entre 1882 y 1892 , es de mayor magnitud con una anomalía anual de -1.32 ${ }^{\circ} \mathrm{C}$, con una mayor contribución también del otoño $\left(-1.5^{\circ} \mathrm{C}\right)$ pero en segundo lugar por el invierno $\left(-1.4^{\circ} \mathrm{C}\right)$ mientras que primavera y verano muestran anomalías menores $(-1.2$ ${ }^{\circ} \mathrm{C}$ y $-1.05{ }^{\circ} \mathrm{C}$ ) respectivamente. Las bajas temperaturas otoñales se deben principalmente a las frías temperaturas diurnas, mientras que el invierno esta más contribuido por las temperaturas nocturnas.

\section{Tabla VI}

TENDENCIAS DECADALES DE LAS SERIES REGIONALES ANUALES Y ESTACIONALES DE LA TEMPERATURA MEDIA, MÁXIMA Y MÍNIMA DIARIA (EN ${ }^{\circ} C$ POR DÉCADA) CALCULADAS PARA EL CONJUNTO DEL PERIODO Y PARA LOS SUBPERIODOS DE CALENTAMIENTO Y ENFRIAMIENTO DETECTADOS. LOS VALORES EN NEGRITA SON SIGNIFICATIVOS AL 0.01. ENTRE PARÉNTESIS EL INTERVALO DE CONFIANZA AL $95 \%$

\begin{tabular}{|c|c|c|c|c|c|c|}
\hline Periodos & Variable & Anual & Invierno & Primavera & Verano & Otoño \\
\hline $1850-2005$ & Tmedia & $\mathbf{0 . 1 0}(0.08 / 0.12)$ & $\mathbf{0 . 1 0}(0.07 / 0.14)$ & $\mathbf{0 . 0 8}(0.05 / 0.12)$ & $\mathbf{0 . 0 9}(0.06 / 0.11)$ & $\mathbf{0 . 1 0}(0.07 / 0.13)$ \\
\hline $1850-2005$ & Tmaxima & $\mathbf{0 . 1 1}(0.09 / 0.14)$ & $\mathbf{0 . 1 2}(0.09 / 0.15)$ & $\mathbf{0 . 1 1}(0.06 / 0.15)$ & $\mathbf{0 . 1 0}(0.06 / 0.13)$ & $\mathbf{0 . 1 2}(0.09 / 0.15)$ \\
\hline $1850-2005$ & Tminima & $\mathbf{0 . 0 8}(0.06 / 0.10)$ & $\mathbf{0 . 0 9}(0.06 / 0.13)$ & $\mathbf{0 . 0 7}(0.04 / 0.09)$ & $\mathbf{0 . 0 8}(0.05 / 0.10)$ & $\mathbf{0 . 0 8}(0.05 / 0.11)$ \\
\hline 1901-1949 & Tmedia & $\mathbf{0 . 2 2}(0.11 / 0.31)$ & $0.10(-0.08 / 0.32)$ & $\mathbf{0 . 2 5}(0.06 / 0.43)$ & $\mathbf{0 . 2 3}(0.07 / 0.38)$ & $\mathbf{0 . 2 6}(0.09 / 0.42)$ \\
\hline 1901-1949 & Tmaxima & $\mathbf{0 . 3 7}(0.25 / 0.46)$ & $0.18(-0.02 / 0.36)$ & $\mathbf{0 . 3 7}(0.16 / 0.60)$ & $\mathbf{0 . 4 4}(0.27 / 0.64)$ & $\mathbf{0 . 4 4}(0.26 / 0.64)$ \\
\hline 1901-1949 & Tminima & $0.08(-0.02 / 0.18)$ & $0.06(-0.15 / 0.24)$ & $0.15(0.01 / 0.31)$ & $0.00(-0.13 / 0.14)$ & $0.09(-0.06 / 0.25)$ \\
\hline 1950-1972 & Tmedia & $-0.19(-0.53 / 0.12)$ & $0.11(-0.58 / 0.68)$ & $-0.52(-1.03 / 0.05)$ & \begin{tabular}{|l|}
$-0.29(-0.71 / 0.13)$ \\
\end{tabular} & $-0.08(-0.57 / 0.53)$ \\
\hline 1950-1972 & Tmaxima & $-0.28(-0.74 / 0.16)$ & $-0.04(-0.61 / 0.62)$ & $-0.62(-1.38 / 0.09)$ & $-0.30(-0.88 / 0.17)$ & $-0.12(-0.84 / 0.70)$ \\
\hline $1950-1972$ & Tminima & $-0.13(-0.51 / 0.14)$ & $0.15(-0.56 / 0.78)$ & $-0.19(-0.72 / 0.29$ & $-0.26(-0.60 / 0.08)$ & $-0.13(-0.41 / 0.33)$ \\
\hline 1973-2005 & Tmedia & $\mathbf{0 . 4 8}(0.36 / 0.66)$ & $0.27(-0.09 / 0.56)$ & \begin{tabular}{|l}
$\mathbf{0 . 7 7}(0.54 / 0.97)$ \\
\end{tabular} & $\mathbf{0 . 6 7}(0.41 / 0.92)$ & $0.29(0.02 / 0.58)$ \\
\hline 1973-2005 & Tmaxima & $\mathbf{0 . 5 1}(0.34 / 0.66)$ & $0.35(0.06 / 0.60)$ & \begin{tabular}{|l|}
$\mathbf{0 . 8 2}(0.53 / 1.15)$ \\
\end{tabular} & \begin{tabular}{|l|}
$\mathbf{0 . 7 3}$ \\
$(0.43 / 1.04)$ \\
\end{tabular} & $0.13(-0.17 / 0.47)$ \\
\hline $1973-2005$ & Tminima & $\mathbf{0 . 4 7}(0.31 / 0.65)$ & $0.06(-0.28 / 0.62)$ & $\mathbf{0 . 6 6}(0.46 / 0.84)$ & $\mathbf{0 . 6 2}(0.38 / 0.93)$ & $\mathbf{0 . 4 3}(0.18 / 0.77)$ \\
\hline
\end{tabular}

En general, podemos hablar de un generalizado incremento térmico del conjunto del territorio español, que abarca desde la segunda mitad del siglo XIX a la actualidad, se manifiesta en la temperatura media diaria pero también en la temperatura mínima diaria y en la temperatura máxima diaria, con tasas de incremento significativas y moderadas, pero mayores que las estimadas para mayores escalas espaciales, y que en los últimos 35 años ha experimentado un incremento notable en sus ratios de calentamiento. 


\section{Bibliografía}

ABAURREA, J., J. ASÍN, O. ERDOZAIN, E. FERNÁNDEZ (2001): Climate variability analysis of temperature series in the Medium Ebro River Basin, en Detecting and Modelling Regional Climate Change, edited by M. Brunet and D. López, pp. 109-118, Springer, New York.

AGUILAR, E., AUER, I., BRUNET, M., PETERSON, T. C., WIERINGA, J. (2003): Guidelines on climate metadata and homogenization. WCDMP-No. 53, WMO-TD No. 1186. World Meteorological Organization, Geneve.

AGUILAR, E., BRUNET, M., SALADIÉ, O., SIGRÓ, J., LÓPEZ, D. (2002): Hacia una aplicación óptima del Standard Normal Homogeneity Test para la homogeneización de series de temperatura. In La información climática como herramienta de gestión ambiental, VII Reunión Nacional de Climatología, Grupo de Climatología de la AGE, Cuadrat JM, Vicente SM, SAZ MA. (eds.). Universidad de Zaragoza: Zaragoza; 17-33.

ALEXANDER, L. V., ET AL., (2006): Global observed changes in daily climate extremes of temperature and precipitation, J. Geophys. Res., 111, D05109, doi:10.1029/2005JD006290.

ALEXANDERSSON, H., MOBERG, A. (1997): Homogenization of Swedish temperature data. Part I: homogeneity test for linear trends. International Journal of Climatology 17: 25-34.

ALMARZA, C. (2000): Respuesta al calentamiento global de la serie de temperatura media anual de Madrid. Actas de la II Asamblea Hispano-Lusa de Geodesia y Geofísica, Lagos (Portugal), Sociedad de Geodesia y Geofísica, Madrid.

ANGOT, A. (1903): Instructions météorologiques, 4ème edition. Gauthier-Villars, Imp-Libraire: Paris.

BARRIENDOS, M., MARTÍN-VIDE, J., PEÑA, J. C., RODRÍGUEZ, R. (2002): Daily Meteorological Observations in Cádiz - San Fernando. Analysis of the Documentary Sources and the Instrumental Data Content (1786-1996), Climatic Change, 53, 151-170.

BRUNET, M., ASIN, J., SIGRÓ, J., BAÑON, M., GARCÍA, F., AGUILA, E., PALENZUELA, J. E., PETERSON, T., JONES, J., (2010): The minimization of the screen bias from ancient Western Mediterranean air temperature records:An exploratory statistical analysis, aceptado por el International Journal of Climatology.

BRUNET, M., SALADIÉ, O., JONES, P., SIGRÓ, J., AGUILAR, E., MOBERG, A., WALTHER, A., LISTER, D., WALTHER, A., ALMARZA, C. (2008): A case-study/guidance on the development of long-term daily adjusted temperature datasets, WMO-TD-1425, Geneva, $57 \mathrm{pp}$.

BRUNET, M., SALADIÉ, O., JONES, P., SIGRÓ, J., AGUILAR, E., MOBERG, A., WALTHER, A., LISTER, D., LÓPEZ, D., ALMARZA, C. (2006): The development of a new daily adjusted temperature dataset for Spain (1850-2003), International Journal of Climatology, 26, 1777-1802, DOI: $10.1002 /$ joc. 1338

BRUNET, M., JONES, P., SIGRÓ, J., SALADIÉ, O., AGUILAR, E., MOBERG, A., DELLA-MARTA, P. D., WALTHER, A., LISTER, D., LÓPEZ, D. (2007): Temporal and spatial temperature variability and change over Spain during 1850-2005, Journal of Geophysical Research-Atmosphere, 112, D12117, doi:10.1029/2006JD008249.

BRUNET, M., E. AGUILAR, O. SALADIÉ, J. SIGRÓ, D. LÓPEZ (2001): A Differential response of Northeastern Spain to asymmetric trends in diurnal warming detected on a global scale, in Detecting and Modelling Regional Climate Change, edited by M. Brunet and D. Lopez, pp. 95107, Springer, New York.

CAUSSINUS, H.; MESTRE, O. (2004): Detection and correction of artificial shifts in climate series. Journal of the Royal Statistical Society. Series C. Applied Statistics, 53: 405-425.

DELLA-MARTA, P. M. Y WANNER, H., (2006): A method of homogenizing the extremes and mean of daily temperature measurements. Journal of Climate, 19(17): 4179-4197.

FENG S., HU Q., QIAN W. (2004): Quality control of daily meteorological data in China, 19512000: a new dataset. International Journal of Climatology 24: 853-870.

GALAN, E., R. CAÑADA, F. FERNÁNDEZ, B. CERVERA (2001): Annual temperature evolution in the southern plateau of Spain from the construction of regional climatic time series, in Detecting and Modelling Regional Climate Change, edited by M. Brunet and D. López, pp. 119-131, Springer, New York. 
GONZÁLEZ, F. J. (1992): El observatorio de San Fernando (1831-1924). Serv. Publ. Ministerio de Defensa: Madrid.

HORCAS, R., D. RASILLA, F. FERNÁNDEZ, (2001): Temperature variations and trends in the Segura River Basin. An exploratory analysis, in Detecting and Modelling Regional Climate Change, edited by M. Brunet and D. López, pp. 133-142, Springer, New York.

JONES, P. D., MOBERG, A. (2003): A hemispheric and large-scale surface air temperature variations: an extensive revision and update to 2001. Journal of Climate 16: 206-223.

JONES, P. D., HULME, M. (1996): Calculating regional climatic time series for temperature and precipitation: methods and illustrations. International Journal of Climatology, 16: 361-377.

KLEIN-TANK, A.M.G., WIKNGAARD, J.B., KÖNNEN, G.P., BÖHM, R., ET AL. (2002): Daily dataset of 20th-century surface air temperature and precipitation series for the European climate assessment. International Journal of Climatology 22: 1441-1453.

MORALES, C. G., M. T. ORTEGA, J. L. LABAJO, A. PIORNO (2005): Recent trends and temporal behavior of thermal variables in the region of Castilla-Leon (Spain), Atmosfera, 18, 71-90.

NCDC (2002): Data documentation for data set 9101, Global Daily Climatology Network, version $1.0,26$ pp. (available online http://www.ncdc.noaa.gov/oa/climate/ climateinventories.html

NICHOLLS, N., TAPP, R., BURROWS, K., RICHARDS, D. (1996): Historical thermometer exposures in Australia. International Journal of Climatology 16: 705-710.

OSBORN, T. J., K. R. BRIFFA, P. D. JONES (1997): Adjusting variance for sample-size in tree-ring chronologies and other regional mean time series, Dendrochronologia, 15, 89-99.

PARKER, D. E. (1994): Effects of changing exposure of thermometers at land stations. International Journal of Climatology 14: 1-31.

SEN, P K. 1968. Estimates of the regression coefficient based on Kendall's tau. Journal of the American Statistical Association, 63: 1379-1389.

SZENTIMREY, T. (1999): «Multiple Analysis of Series for Homogenization (MASH)», Proceedings of the Second Seminar for Homogenization of Surface Climatological Data, Budapest, Hungary; WMO, WCDMP-No. 41, pp. 27-46

TAN, L. S., BURTON, S., CROUTHAMEL, R., VAN ENGELEN, A., HUTCHINSON, R., NICODEMUS, L., PETERSON, T. C., RAHIMZADEH, F. (2004): Guidelines on Climate Data Rescue. WMO/TD 1210, WCDMP-55, Geneva: $12 \mathrm{pp}$.

TREWIN, B.C. (1999): The development of a high quality daily temperature data set for Australia, and implications for the observed frequency of extreme temperatures. In: Meteorology and oceanography at the new millennium: AMOS 1999. Proceedings of the Sixth National Australian Meteorological and Oceanographic Society Congress, Canberra, 1999.

VINCENT, L. A., ZHANG, X., BONSAL, B. R., HOGG, W. D. (2002): Homogenization of daily temperatures over Canada. Journal of Climate 15: 1322-1334.

VOSE, R. S., D. R. EASTERLING, AND B. GLEASON, (2005): Maximum and minimum temperature trends for the globe: An update through 2004, Geophys. Res. Lett., 32, L23822, doi:10.1029/2005GL024379.

ZHANG, X., L. A. VINCENT, W. D. HOGG, A. NIITSOO (2000): Temperature and precipitation trends in Canada during the 20th century, Atmos. Ocean, 38, 395-429. 
